\title{
Idiopathic Hemi facial Atrophy: A Rare Case Report
}

\author{
Dr. Mukta Pagrani ${ }^{1}$, Dr. Chander Mohan ${ }^{2}$, Dr. Manshu Singla ${ }^{3}$, \\ Dr. Abhinav Srivastava ${ }^{4}$ \\ ${ }^{1}$ Assistant Professor, Dept. of ENT, Rohilkhand Medical College \& Hospital, Bareilly, India \\ ${ }^{2}$ Professor and Head, Dept. of ENT, Rohilkhand Medical College \& Hospital, Bareilly, India \\ ${ }^{3} P G 2^{\text {nd }}$ Year, Dept. of ENT, Rohilkhand Medical College \& Hospital, Bareilly, India \\ ${ }^{4}$ Assistant Professor, Dept. of ENT, Rohilkhand Medical College \& Hospital, Bareilly, India
}

\begin{abstract}
Idiopathic hemifacial atrophy also known as Parry-Romberg syndrome (PRS) is a rare neurocutaneous syndrome. It is characterized by slowly progressive atrophy of one side of the face, usually involving the skin, fat and connective tissue. A rare case of Parry-Romberg syndrome in a 30 year-old female is presented because of its rarity. The diagnosis of Parry-Romberg syndrome as established mainly based on the clinical findings. Not much is reported about the efficacy of agents used in the treatment of this syndrome in the literature making treatment decisions very difficult.
\end{abstract}

Keywords: Hemifacial atrophy, Parry-Romberg syndrome(PRS),Trigeminal Neuralgia.

\section{Introduction}

Parry-Romberg syndrome was described by Parry in 1825 and by Romberg in 1846, by naming it Prophoneurosis. Eulenberg termed it 'progressive facial hemi atrophy' in 1871(1).This rare disease is characterized by atrophy of the skin, fat, connective tissue and muscles of one side of the face and so is also called idiopathic progressive hemifacial atrophy. This is an acquired condition of unknown aetiology presenting usually in childhood, with gradual progression over the years after which stabilization occurs The incidence is higher in females. Patients with earlier onset of the disease are often more severely affected, with involvement of facial cartilage and bones. Once the disease is established it does not exhibit spontaneous improvement $(2,3,4)$. Occasionally, there may be some neurological complications, such as trigeminal neuralgia, facial paresthesia, severe headache and contralateral epilepsy. Neuropsychiatric problems are common in ParryRomberg syndrome patients with reported prevalence as high as $50 \%$.

\section{Case Report}

A 30-year-old female presented with complaints of left sided facial atrophy of 5 yrs duration, along with headache and on and off facial pain, which were intermittent, sharp and of stabbing nature. She had no history of fever, convulsions, loss of consciousness or ear, nose, throat problem. The patient was moderately built and nourished. General, physical and systemic examination were normal. Right side of the face appeared normal. There was clinically evident hemifacial wasting on left side. There was no enophthalmos, lid retraction or ocular abnormalities on ophthalmologic consultation. There were no neurological symtoms. Left side of the face showed signs of hemi atrophy (Fig. 1, 2, and 3). Examination of the face revealed no sensory or motor deficits on both sides. There was no evidence of Horner's syndrome, facial palsy or hemi facial spasm. Measurement were taken from the nasion to the tragus, nasion to angle of mandible, and mid chin to tragus of both right and left side .The measurements showed hemi facial atrophy of the left side [Table 1]. Routine blood and urine investigations were within normal limits, Diagnostic radiological imaging was done in which ultrasound abdomen (USG abdomen) showed no organomegaly or free fluid. X-ray chest and skull were normal. Treatment for facial pain was started with Carbamezipine $200 \mathrm{mg}$ three times a day along with calcipotriolbetamethasone ointment for local application. she showed remarkable improvement in five days. This treatment was continued for 1 month and then gradually tapered and stopped. At the end of treatment, there were no symptoms of pain, though facial hemiatrophy persisted. Patient is under regular follow-up for 1 year and has not shown any recurrence of pain.

Table 1.

\begin{tabular}{|c|c|l|c|c|c|}
\hline \multicolumn{2}{|l|}{ Nasion to the Tragus } & \multicolumn{2}{l|}{ Nasion to Angle of Mandible } & \multicolumn{2}{l|}{ Mid chin to Tragus } \\
\hline Right & Left & Right & Left & Right & Left \\
\hline $13.5 \mathrm{cms}$ & $13.9 \mathrm{cms}$ & $14 \mathrm{cms}$ & $15 \mathrm{cms}$ & $16 \mathrm{cms}$ & $16.5 \mathrm{cms}$ \\
\hline
\end{tabular}




\section{Discussion}

Parry Romberg syndrome manifests in the first or second decade of life with a slow progression over many years showing atrophy and then becomes stable. Alterations concerning involvement, duration and deformity can stabilize in any stage of growth and development $(5,6)$.

The aetiology of Parry-Romberg syndrome remains unknown, although various theories have been proposed. These include the trigeminal theory that considers the wasting process as result of trauma to the superior cervical ganglia(2). The autoimmune hypothesis has been proposed due to the observation of inflammatory changes and the presence of autoimmune antibodies. Recently extracutaneous involvement has been described in literature raising awareness that this is not only a cutaneous disease $(3,4)$. The clinical features include apart from the hemifacial atrophy, hemiatrophy of the contralateral or ipsilateral arm, trunk or leg. Dental and ocular abnormalities are not rare with enophthalmos, uveitis, episcleritis \& ocular myopathy. The eye usually works normally and the ears can be smaller than normal ones, due to the atrophy(5). Most commonly articular manifestations observed are arthritis and neurological abnormalities presenting mainly with headaches, migraine, facial pain, and seizures(3,4). Central Nervous System(C.N.S.) involvement seems to occur more often in patients with Parry-Romberg syndrome, most commonly consisting of migraine-type headache, focal epilepsy and trigeminal neuralgia.

A recent global internet survey of 205 patients with Parry-Romberg syndrome estimated the central nervous system involvement to be over fifty percent(7). In the present case there was no involvement of limbs, trunk, oral cavity, eye and CNS. The extension of the atrophy is frequently limited to one side of the face, and the ipsilateral involvement of body is rare.

It is a self-limiting condition and there is no definitive cure. Patients, who manifest atrophy in early ages, have a better outcome. Various systemic treatments have been tried for Parry-Romberg syndrome including oral steroids, D-penicillamine, antimalarials, methotrexate, cyclophosphamide, cyclosporine, and azathioprine(3). These aggressive immunosuppressive treatments are chosen based mainly on the disease activity as well as the extracutaneous complications. There is no standard treatment regimen for Parry-Romberg syndrome. Both topical steroids and Vit D3 analogues that are proposed to be used for uncomplicated localised scleroderma could be beneficial, as they both prevent fibroblast proliferation and have anti-inflammatory action. In case of early diagnosis, consider initiation of early treatment in order to try preventing the disease evolution. The response to treatment for Parry-Romberg syndrome is very difficult to evaluate though and no trials are yet available to compare a drug's safety and efficacy in this disease. Over the years, plastic surgery has come up in a high way for confronting the severe disfigurement caused with very good cosmetic results, using fat, silicon and bone implants, flap/pedicle grafts(3) and recently cell fat mixed with platelet gel(8).

\section{Conclusion}

Although there has been considerable improvement in the diagnosis and management of Parry Romberg syndrome and its extracutaneous manifestations, there are no standard definitive guidelines to be followed for treating the disease. A valid treatment protocol needs to be developed in order to establish the best treatment approach for this rare but severely disfiguring disease.

\section{References}

[1]. Goldhammer Y, Kronenberg J, Tadmor R, Braham J, Leventon G: Progressive hemifacial atrophy (Parry-Romberg's disease), principally involving bone.J Laryngol Otol 1981, 95:643

[2]. Gulati S, Jain V, Garg G. Parry Romberg syndrome. Indian J Pediatr. 2006;73:448-449.

[3]. Stone J. Parry-Rombergsyndrome. PracticalNeurology. 2006;6:185-188. 4.Tollefson MM, Witman PM. En coup de sabre morphea and Parry-Romberg syndrome: a retrospective review of 54 patients. J Am Acad Dermatol. 2007;56:257-263.

[4]. Mazzeo N, Fisher JG, Mayer MH, Mathieu GP, Mcade FGG: Progressive hemifacial atrophy (Parry Romberg Syndrome)

[5]. OralSurgOralMedOralPatholOralRadiolEndod 1995, 79:30-35.

[6]. Roddi R, Riggio E, Gilbert PM, Houvius SER, Vaandrager JM, van derMeulen JCH: Clinical evaluation of techiniques used in the surgical treatment of progressive hemifacial atrophyJ Craniomaxilofac Surg 1994, 22:23-32.

[7]. Stone J. Parry-Romberg syndrome: a global survey of 205 patients using the Internet. Neurology.2003;61:674-676.

[8]. Cervelli V, Gentile P. Use of cell fat mixed with platelet gel in progressive hemifacial atrophy.Aesthetic Plast Surg. 2009;33:22-27. 


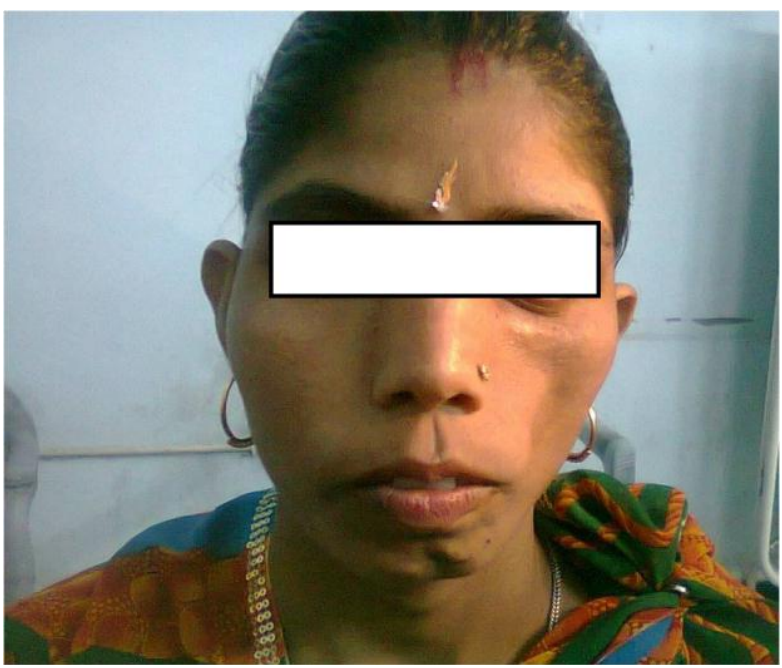

Figure 1: View from front

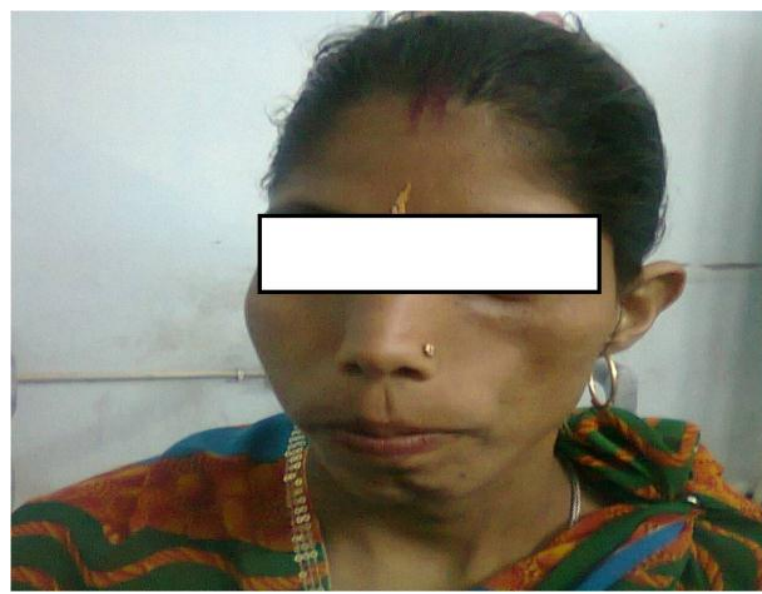

Figure 2: View from left oblique

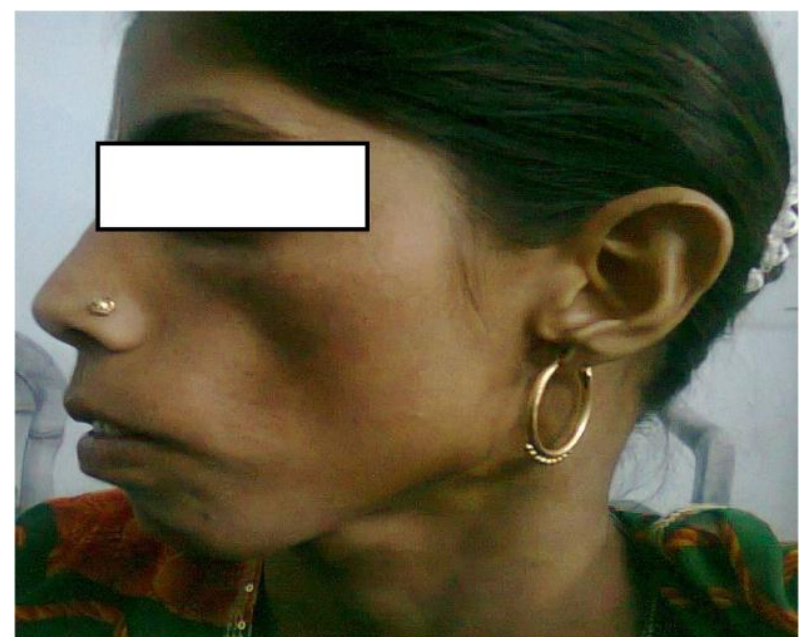

Figure 3: View from left All view showing left sided hemifacial atrophy 